\title{
Research of Numerical Simulation on Perforation of Conical Nose Rigid Projectile into Alluminum Alloy Target
}

\section{Xu Ruihuai}

School of Mechanics and Photoelectric Physics, Anhui University of Science and Technology, Huainan, China

\section{Email address:}

rhxu@x263.net

\section{To cite this article:}

Xu Ruihuai. Research of Numerical Simulation on Perforation of Conical Nose Rigid Projectile into Alluminum Alloy Target. Science Discovery. Vol. 5, No. 1, 2017, pp. 7-11. doi: 10.11648/j.sd.20170501.12

Received: January 19, 2017; Accepted: March 9, 2017; Published: March 15, 2017

\begin{abstract}
In this paper,the penetration and perforation process of the conical nose rigid projectile into alluminum alloy target were simulated numerically by using FEM. Two sets of the projectiles and targets in different sizes were used in calculation models. The first set took the same size as the tested projectile and target for comparison, the second set changed diameter of projectile to evaluate its influence on the perforation capacity. The residual velocity were obtained by using numerical simulation. The simulation results indicate that in the same conditions, the calculated residual velocity coincides approximately with the existing data. It shows the validity and exactness of the proposed model. The projectile with smaller diameter possess better perforation performance.
\end{abstract}

Keywords: Conical Nose Projectile, Perforation, Residual Velocity, Numerically Simulation

\section{锥头刚性弹贯穿铝合金靰数值模拟研究}

许瑞淮

力学与光电物理学院, 安徽理工大学, 淮南, 中国

邮箱

rhxu@x263. net

摘要：对具有圆雉形头部的刚性杆式弹侵彻和贯穿铝合金靶板进行了有限元数值模拟分析。弹和靶采用二种尺寸:第 一种尺寸与实验的弹和靶尺寸相同; 第二种尺寸考虑了弹径的微小变化对其贯穿靶的影响。通过数值模拟, 获得了弹体 贯穿靶后的剩余速度。计算结果表明, 在同样条件下, 弹贯穿靶后剩余速度与已有的结果基本吻合, 说明本文计算模型是 有效和精确的。并且, 弹径变小, 其侵彻、贯穿靶的威力将有所提高。

关键词: 锥头弹, 贯穿, 剩余速度, 数值模拟

\section{1. 引言}

目前在应对突发载荷、意外袭击及敌方攻击的弹道防 护中, 人们对铝合金在轻质防护结构设计中的作用具有日 益增长的特殊兴趣 [1] [2] [3] [4]。尽管如此, 对这类问题 进行详细理论、数值模拟和实验研究的报道仍相当有限。
F o r r e s t a 1等 [2] 将靶体视为不可压缩幕次 硬化材料, 基于空腔膨胀理论建立了尖雉 (卵) 头弹体贯穿 金属靶体的理论模型。Ch e n X i a ow e i 等 [5]忽略 靶背自由表面效应的影响, 考虑了靶体材料的可压缩性, 分别建立了刚性尖头弹贯穿理想弹塑性和幂次硬化靶体 的分析模型。We n Hem i n g [6] 基于半经验公式得到 
了弹体贯穿不同材料靶体的终点弹道公式, 同样也忽略了 靶背自由表面效应的影响。

目前对动能弹高速侵彻和贯穿问题的研究方法中, 现 场实验研究必不可少, 但耗资巨大, 费时费力; 而分析模 型往往有过多的物理假定, 由于穿甲问题的复杂性, 使理 论分析十分困难; 数值方法逐渐成为研究高速侵彻贯穿方 面的主要方法。

本文基于MSC. DYTRAN软件对具有圆锥形头部的刚性 杆式铇弹侵彻和贯穿铝合金靶板过程进行了有限元数值 模拟分析。弹和靶采用两种尺寸: 第一种尺寸与已有实验 ${ }^{[3]}$ 的弹尺寸相同, 以便将计算结果和实验结果进行比较; 第二种尺寸考虑弹径微小变化的影响, , 具体分 3 种弹径进 行计算。针对第一种尺寸弹体, 模拟撞击的是实验使用的 $12.7 \mathrm{~mm} 、 50.8 \mathrm{~mm}$ 和 $76.2 \mathrm{~mm}$ 厚 $5083-\mathrm{H} 131$ 铝合金靶和 $25.4 \mathrm{~mm}$ 厚6061-T651铝合金靶。针对第二种尺寸弹体，模拟撞击 的是实验使用的 $12.7 \mathrm{~mm} 、 50.8 \mathrm{~mm}$ 和 $76.2 \mathrm{~mm}$ 厚 $5083-\mathrm{H} 131$ 铝 合金靶。靶为边长 $20 \mathrm{~cm}$ 的正方形板, 弹以一定的初始速度 正撞击靶体。通过数值模拟, 获得了弹体贯穿靶后剩余速 度、弹的速度时程曲线及弹径的变化对其贯穿靶威力的影 响。

为简单计，在本文模拟计算中，不考虑弹靶之间的摩 擦效应, 不考虑应变率效应及冲击绝热温升对靶材料本构 关系的影响。

\section{2. 弹靶模型}

\section{1. 弹的模型}

锥头弹的几何形状如图1所示, 弹体头部为圆雉形, 后部为圆柱形。弹后部长度为 $L$, 圆雉头部长度为 1 , 雉角为 $2 \phi$ 。由实验观察 [3] [4], 弹初始速度不超过一定范围 $(1200 \mathrm{~m} / \mathrm{s})$ 时, 弹贯穿靶后用肉眼看不出有变形, 故计 算时设置为刚体，采用Dytran中的刚体材料本构模型 (MATRIG) [7]。

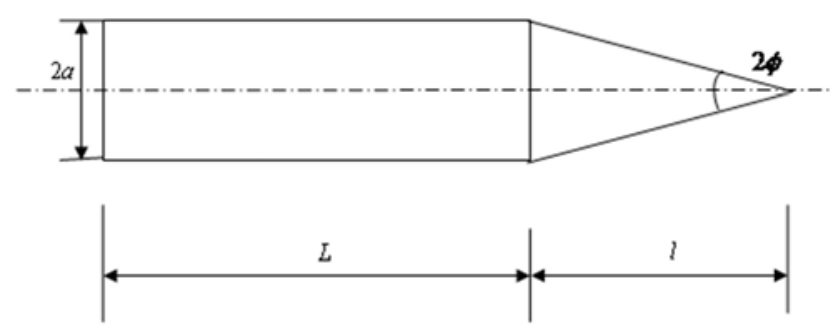

图1 弹体的几何形状。

\section{2. 靶的模型}

靶材料为5083-H131铝合金和6061-T651铝合金, 采用 Dytran中的硬化弹塑性材料本构模型 (DYMAT24)。由单轴 大应变压缩实验数据拟合的 5083-H131 铝合金和 6061-T651铝合金的真应力-真应变关系为幕函数形式, 如 下式所示。

$$
\sigma=\left\{\begin{array}{cc}
E \varepsilon, & \sigma<Y \\
Y\left(\frac{E \varepsilon}{Y}\right)^{n}, & \sigma \geq Y
\end{array}\right.
$$

上式中, $\sigma$ 为真应力, $\varepsilon$ 为真应变, $E$ 为杨氏模量, $Y$ 为初始屈服应力, $\mathrm{n}$ 为应变硬化指数。对 $5083-\mathrm{H} 131$ 铝合金, $\mathrm{E}$ 为 $70.3 \mathrm{GPa}, \mathrm{Y}$ 为 $276 \mathrm{MPa}, \mathrm{n}$ 为 0.084 ; 对6061-T651铝合金, $\mathrm{E}$ 为 $69 \mathrm{GPa}, \mathrm{Y}$ 为 $262 \mathrm{MPa}, \mathrm{n}$ 为 0.085 。

5083-H131铝合金和6061-T651铝合金的真应力-真应 变曲线比较图如图2所示 $[8]$ 。

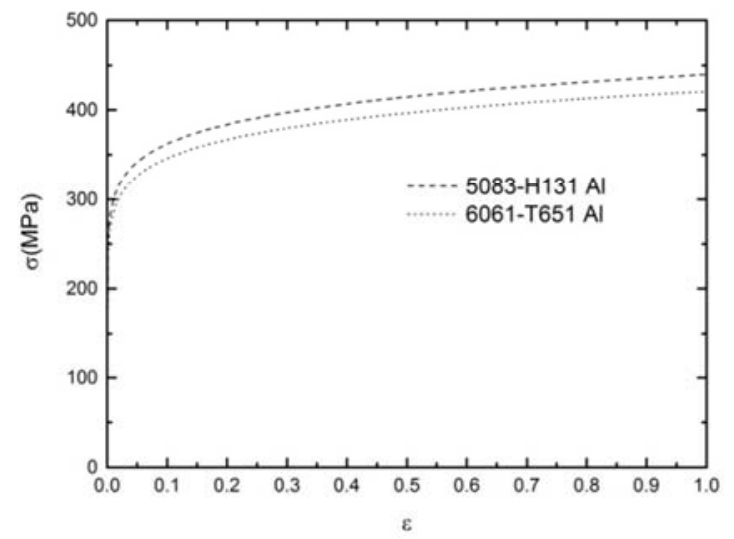

图2 5083-H131铝合金和6061-T651铝合金的真应力-真应变曲线。

\section{3. 弹靶的有限元模型}

弹靶初始有限元划分如图3、图4、图5和图6所示。对 图3、图 4 和图 5 , 弹材料为铇合金, 密度为 $18500 \mathrm{~kg} / \mathrm{m}^{3}$, 几何尺为: $2 \mathrm{a}=8.31 \mathrm{~mm}, \mathrm{~L}=20.7 \mathrm{~mm},, 1=14.8 \mathrm{~mm}$, 弹质量为 $26 \mathrm{~g}$, 靶材料为 $12.7 \mathrm{~mm} 、 50.8 \mathrm{~mm}$ 和 $76.2 \mathrm{~mm}$ 厚 $5083-\mathrm{H} 131$ 铝合 金; 对图6, 弹材料为钢, 密度为 $8100 \mathrm{~kg} / \mathrm{m}^{3}$, 几何尺为: $2 \mathrm{a}=9.53 \mathrm{~mm}, \mathrm{~L}=131.7 \mathrm{~mm},, 1=14.3 \mathrm{~mm}$, 弹质量为 $79 \mathrm{~g}$, 靶材 料为 $25.4 \mathrm{~mm}$ 厚6061-T651铝合金。

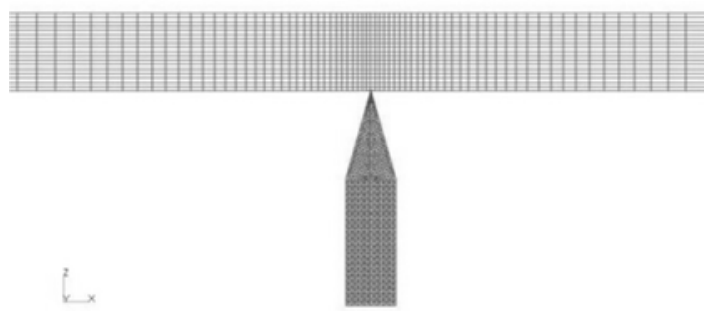

图3 弹靶初始有限元划分（12.7mm厚5083-H131铝合金靶）。

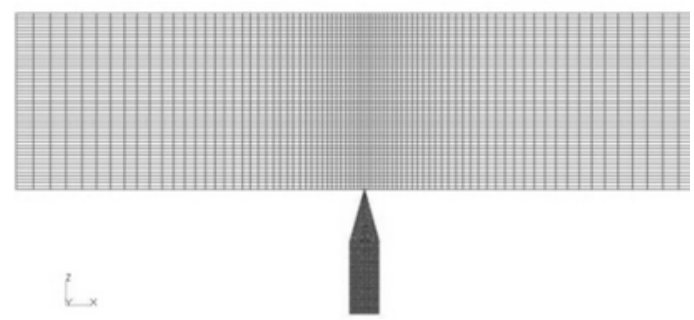

图4 弹靶初始有限元划分（50.8mm厚5083-H131铝合金靶）。 


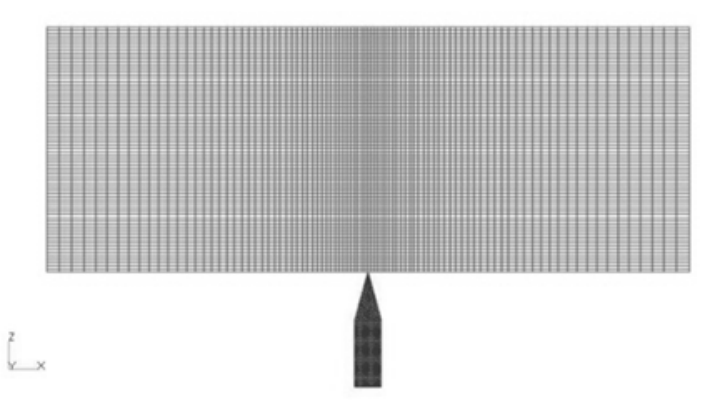

图5 弹靶初始有限元划分（76.2mm厚5083-H131铝合金靶）。

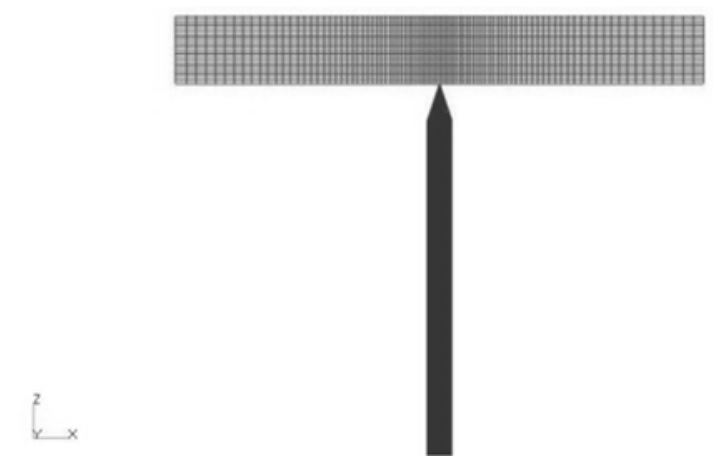

图6 弹靶初始有限元划分（25.4mm厚6061-T651铝合金靶）。

对刚性弹体，采用 4 节点四面体单元划分单元，单元 尺度为 $0.5 \mathrm{~mm}$ 。

对靶体, 采用8节点六面体单元划分单元, 沿厚度方 向均匀划分单元（单元尺度为 $1 \mathrm{~mm}$ ）。为保证计算精度及 减少单元数目, 沿边长按等比方式非均匀划分单元, 中间 (弹靶接触区域) 密, 两边疏, 两边单元尺度为 $4 \mathrm{~mm}$, 中 间单元尺度为 $1 \mathrm{~mm}$ 。

在刚性弹侵彻铝合金靶板过程中, 如果靶中某单元的 等效塑性应变达到 1.5 , 则认为该单元被侵蚀掉, 将该单 元删除。

在高速碰撞等问题的计算中，可采用对称罚函数法处 理碰撞-接触界面。罚函数值的大小受到稳定条件的限制。 若计算中发生明显穿透, 可放大罚函数值或缩小时间不长 来调节 [9] [10]。

对于弹侵彻靶体过程中的大畸变单元, 采用侵蚀法处 理。这种方法是将交界面上的大畸变单元去掉，而这些大 畸变单元往往处于侵蚀孔底附近的高压、高应变区域，大 畸变单元去除后形成空穴和压力释放, 没有侵蚀掉的单元 又于弹体进行接触和碰撞, 产生大畸变后又被侵蚀去掉。 这样就把一个连续的侵彻过程变成了一系列间断的撞击 $[11][12]$ 。

\section{4. 数值计算结果}

图7、8、9和10为弹撞击靶的典型侵彻图像。

图11、12、13和 14 为弹的剩余速度与已有实验的比较。 结果表明, 数值计算的弹贯穿靶后剩余速度与已有结果基 本吻合, 说明本文数值模拟计算是有效和精确的。

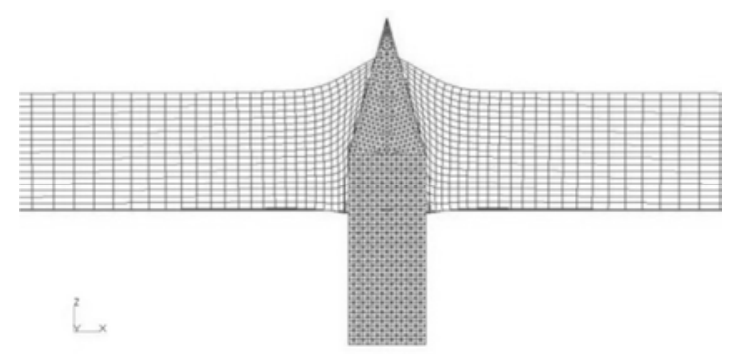

图7 初始速度为 $600 \mathrm{~m} / \mathrm{s}$ 弹撞击 $12.7 \mathrm{~mm}$ 厚靶的典型侵彻图像 $(\mathrm{t}=36.04 \mu \mathrm{s}$, Cycle $=1500$ )。

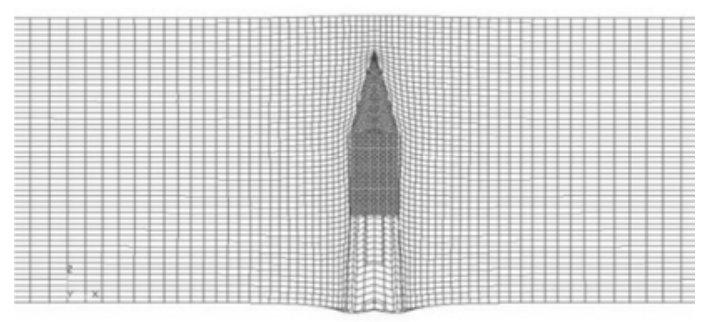

图8 初始速度为 $1100 \mathrm{~m} / \mathrm{s}$ 弹撞击 $50.8 \mathrm{~mm}$ 厚靶的典型侵彻图像 $(\mathrm{t}=43.46 \mu$ $\mathrm{s}, \mathrm{Cycle}=1500)$ 。

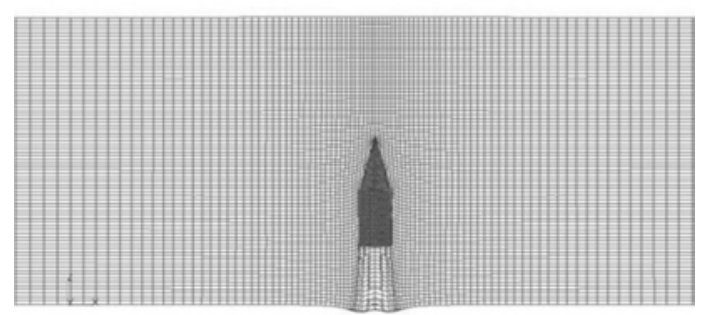

图9 初始速度为 $1100 \mathrm{~m} / \mathrm{s}$ 弹撞击 $76.2 \mathrm{~mm}$ 厚靶的典型侵彻图像 $(\mathrm{t}=43.19 \mu$ $\mathrm{s}, \mathrm{Cycle}=1500)$ 。

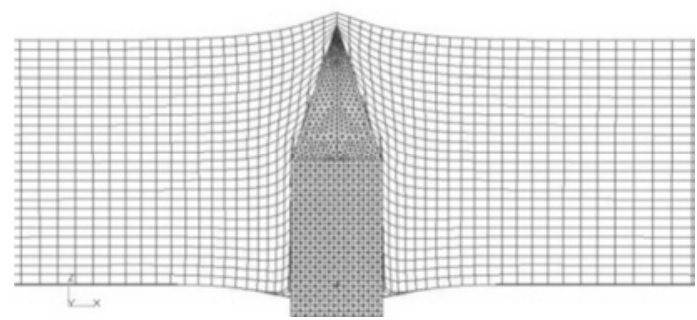

图 10 初始速度为 $600 \mathrm{~m} / \mathrm{s}$ 弹撞击 $25.4 \mathrm{~mm}$ 厚靶的典型侵彻图像 (局部) $(\mathrm{t}=46.53 \mu \mathrm{s}, \mathrm{Cycle}=1500)$ 。

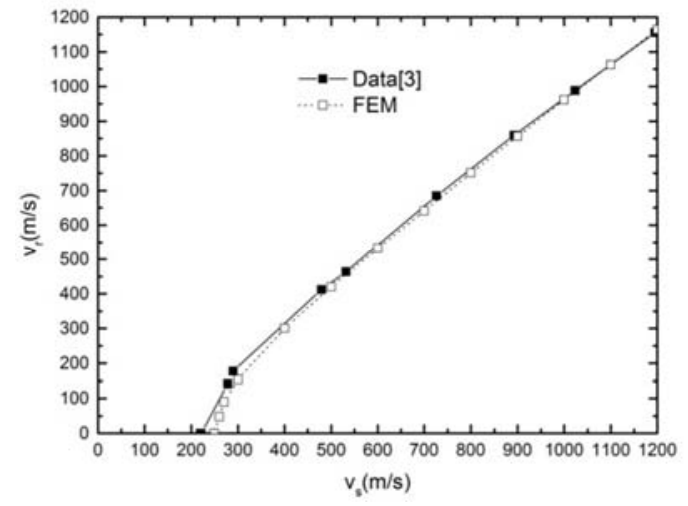

图11 弹的剩余速度与已有实验的比较（5083-H131铝靶, 厚 $12.7 \mathrm{~mm})$ 。 


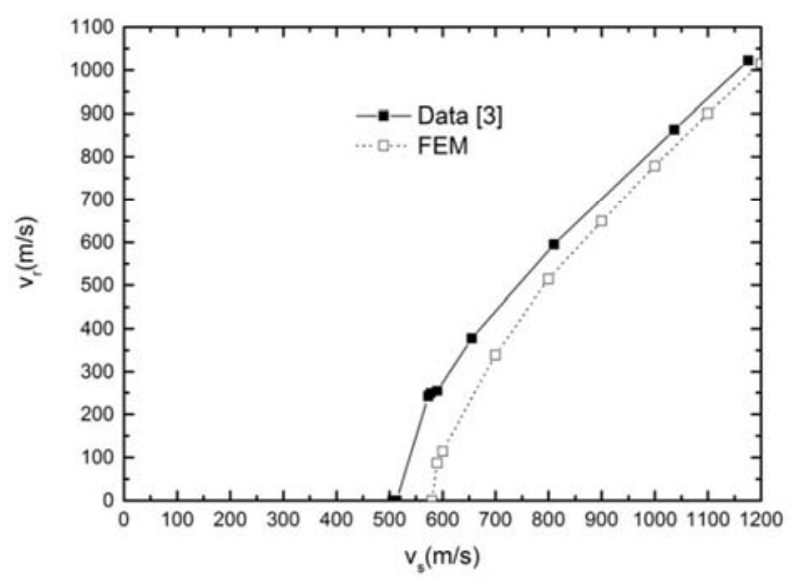

图12 弹的剩余速度与已有实验的比较 (5083-H131铝靶, 靶厚 $50.8 \mathrm{~mm}$ )。

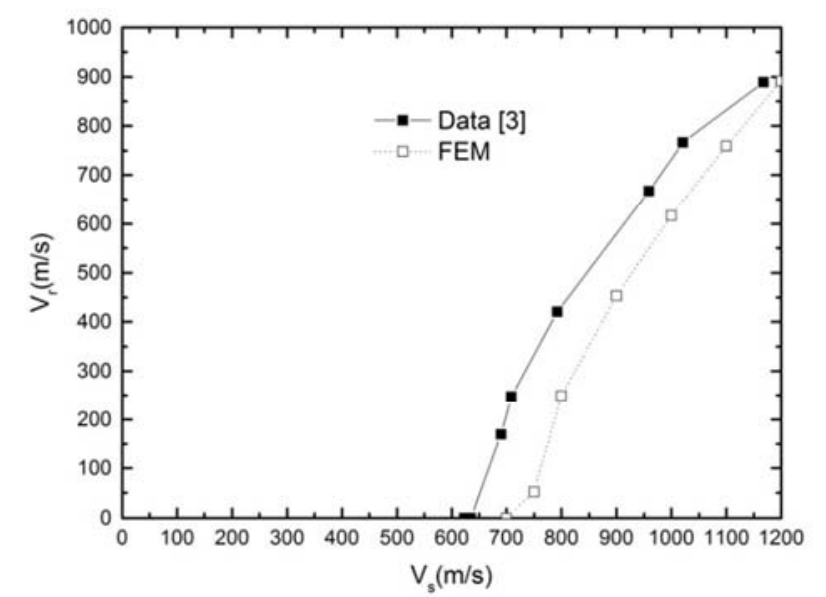

图13 弹的剩余速度与已有实验的比较 (5083-H131铝靶, 靶厚 $76.2 \mathrm{~mm}$ )。

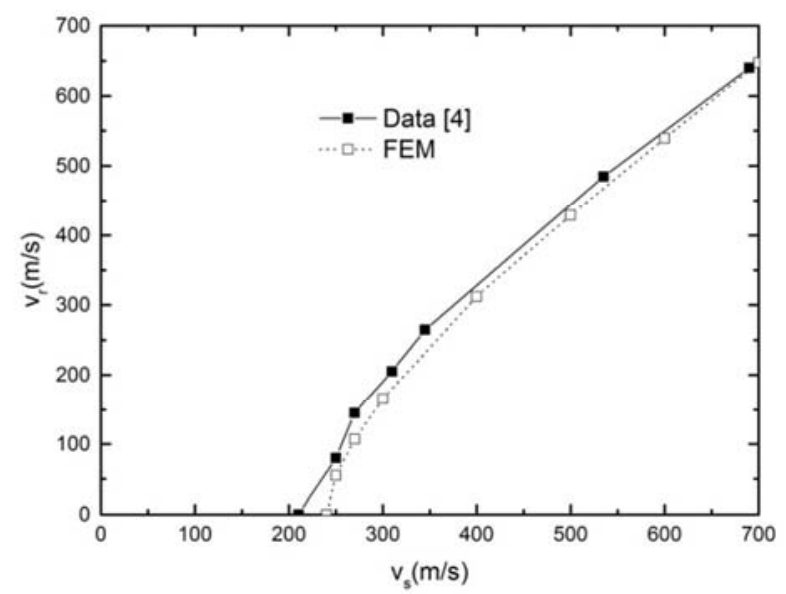

图14 弹的剩余速度与已有实验的比较 (6061-T651铝靶, 靶厚 $25.4 \mathrm{~mm}$ )。

\section{5. 考虑弹径变化对弹体贯穿的影响}

为了考虑弹径变化的影响, 在保持弹质量和1不变的 情况下, 将a进行微小变化, 本文共考虑 3 种弹径, 其中弹 径居中者即为前文中的实验尺寸弹，模拟撞击是前文中的 三种厚度的5083-H131铝合金靶。

图15、16和17为在各种初始速度下考虑不同弹径弹侵 彻三种厚度的5083-H131铝合金靶的剩余速度比较图。计
算结果表明, 在同样条件下, 弹径越小，弹的剩余速度越 大。

图18、19和20为考虑不同弹径弹的典型速度时程曲线。 从图中可以看出, 在同样条件下, 弹径越小, 弹侵彻三种 厚度的5083-H131铝合金靶板过程中的阻力或减加速度越 小，弹的剩余速度越大。

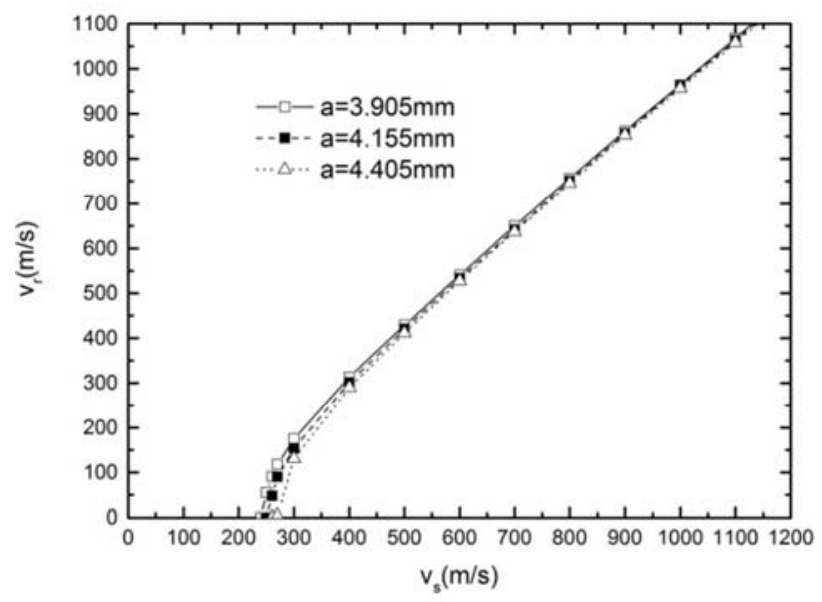

图15 不同弹径弹剩余速度比较（12.7mm厚5083-H131铝合金靶）。

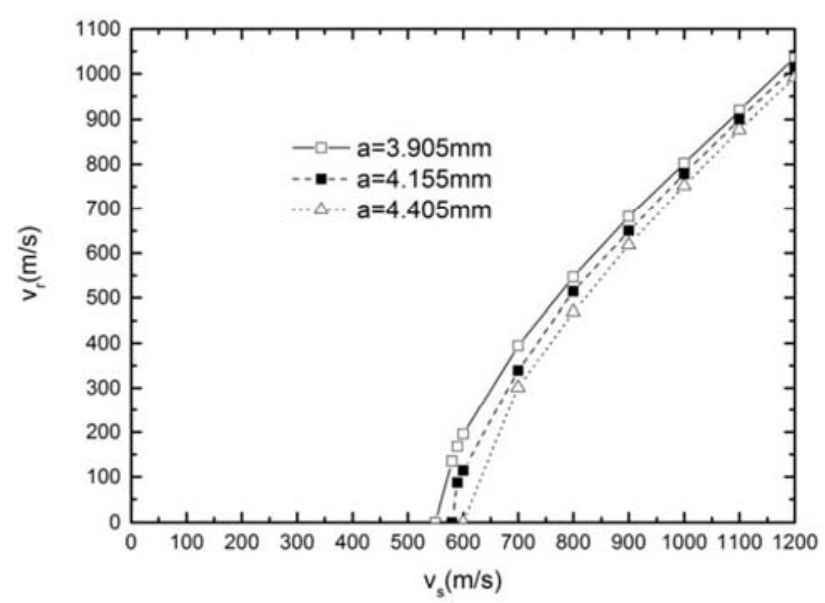

图16 不同弹径弹剩余速度比较（50.8mm厚5083-H131铝合金靶）。

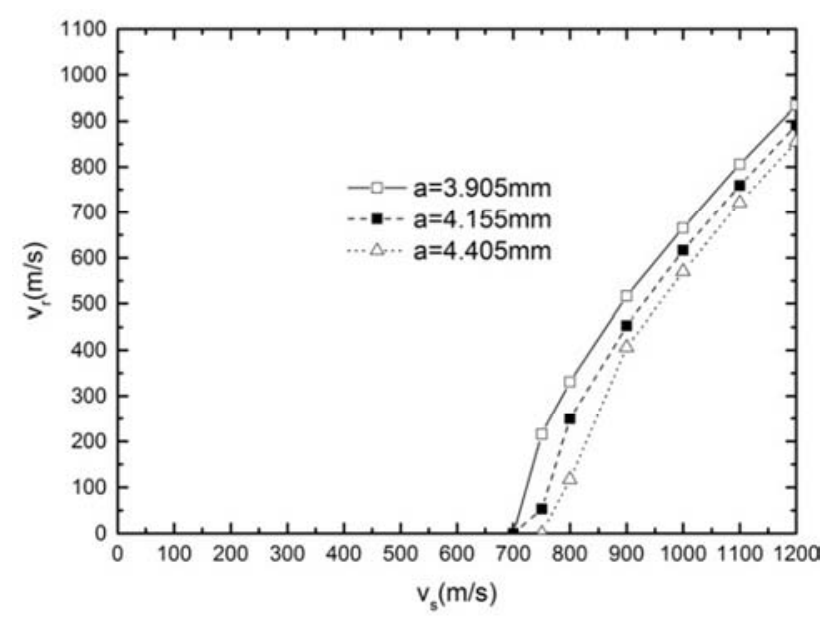

图17 不同弹径弹剩余速度比较（76.2mm厚5083-H131铝合金靶）。 


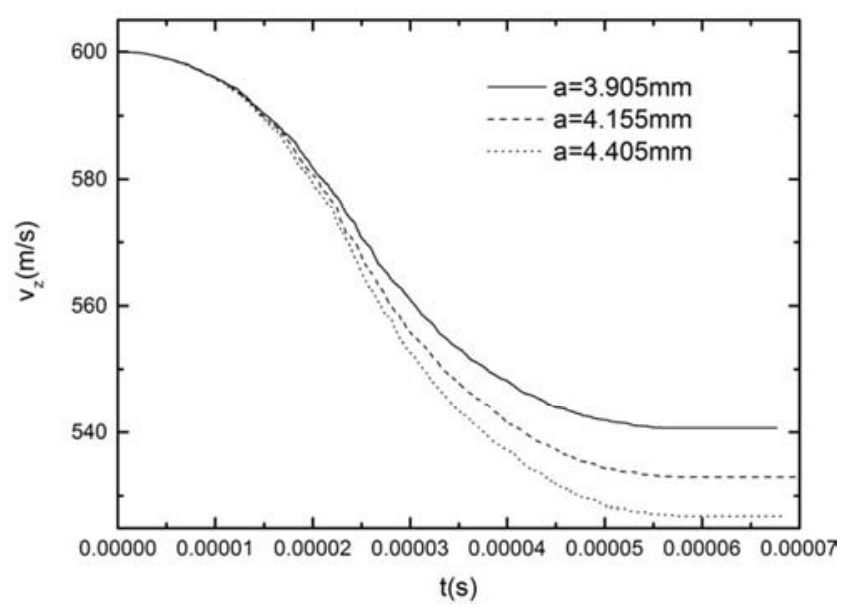

图18 不同弹径弹的典型速度时程曲线 (12.7mm厚5083-H131铝合金靶)。

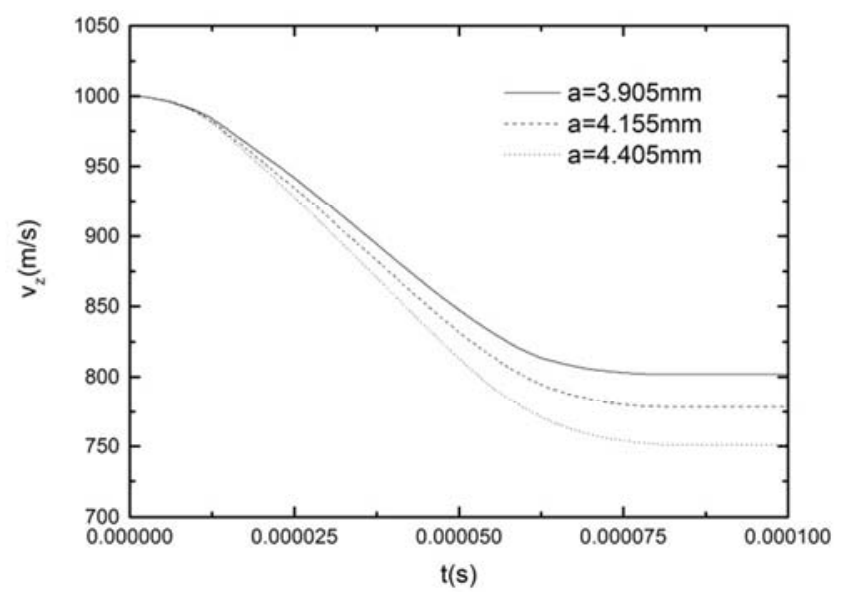

图19 不同弹径弹的典型速度时程曲线 (50.8mm厚5083-H131铝合金靶)。

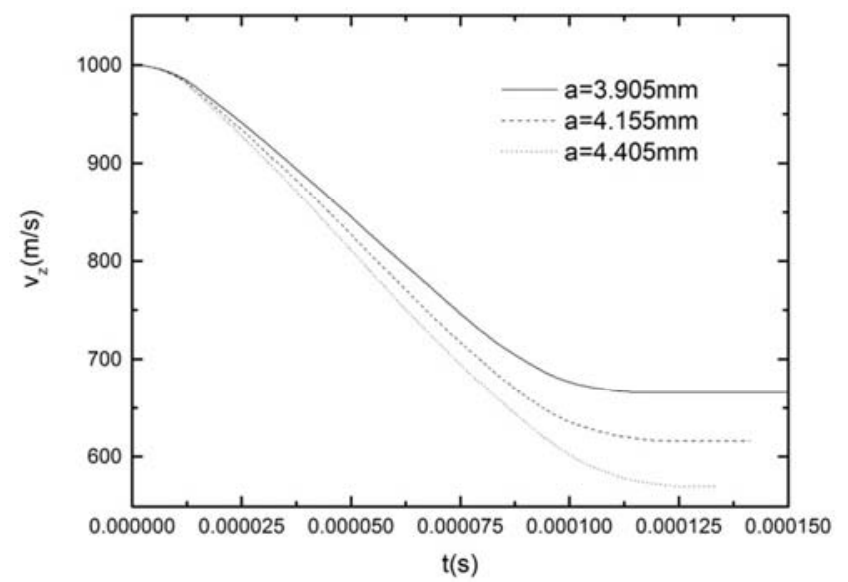

图20 不同弹径弹的典型速度时程曲线 (76.2mm厚5083-H131铝合金靶)。

\section{6. 结论}

本文对具有圆雉形头部的刚性弹侵彻和贯穿铝合金 靶板进行了有限元数值模拟分析。通过数值模拟, 获得了 弹体贯穿靶后剩余速度、弹的速度时程曲线和弹径的变化
对其贯穿靶威力的影响。计算结果表明, 在同样条件下, 弹贯穿靶后剩余速度与已有的实验结果相吻合, 说明本文 计算模型是有效和精确的。并且弹径变小, 其侵彻、贯穿 靶的威力将有所提高。本文计算模型和结果对相关的工程 计算具有一定的参考价值。

\section{参考文献}

[1] T. Børvik, M. J. Forrestal, 0. S. Hopperstad, et al. Perforation of AA5083-H116 aluminium plates with conical-nose steel projectiles-Calculations $[J]$. Int J Impact Eng, 2009, 36: 426-437.

[2] M. J. Forrestal, T. L. Warren. Perforation equations for conical and ogival nose rigid projectiles into aluminum target plates[J]. Int J Impact Eng, 2009, $36: 220-225$.

[3] M. J. Forrestal, V. K. Luk, N. S. Brar, Perforations of aluminum armor plates with conical-nose projectiles[J]. Mech Mater, 1990, 10: 97-105.

[4] Forrestal MJ, Rosenberg Z, Luk VK, Bless SJ. Perforations of aluminum plates with conical-nosed projectiles. J Appl Mech 1987; 54:230-2.

[5] Chen Xiaowei, Li Qingming. Perforationof a thick plate by rigid projectiles[J]. International Journal of Impact Engineering, 2003, 28(7) :743-759.

[6] Wen Heming. Predicting the penetration and perforation of targets by projectile at normal incidence $[\mathrm{J}]$. Mechanics of Structures and Machines, 2002, $30(4): 543-577$.

[7] 丁沛然, 钱纯. 非线性瞬态动力学分析-MSC. Dytran理论及 应用 [M]. 北京: 科学出版社, 2006:56-9。

[8] Kawahara W. Compression testing of materials at low-to-medium strain rates[R]. Paper no. 86-WA-Mats-15, presented at the ASME Winter Annual Meeting, Anaheim, CA 1986.

[9] 罗琳, 邢政权. 弹丸侵彻混凝土靶的MSC. DYTRAN数值模拟 $[J]$, 沈阳理工大学学报 [J].2012, 31（6）: 73-76。

[10］许瑞淮，聂国华，黄志强. 尖头刚性钨弹贯穿铝合金靶的 数值模拟分析 $[J]$, 兵工学报, 2010,31 (增刊1) : 140-143。

[11] Qiaoguo WU, Heming Wen. Petalling of a thin metal plate struck by a conical-nosed projectile[J]. Acta Mechanica Solida Sinica, 2015. 10, 28(5) : 568-577.

[12] 肖云凯, 方秦, 吴昊等. 考虑靶背自由表面和开裂影响的刚 性尖头弹贯穿金属靶板模型 $[\mathrm{J}]$. 爆炸与冲 击, 2016. 5, 36 (3) :359-369。 